\title{
IOT based Automated Intrusion Detection System
}

\author{
Md. Yousuf Hossain \\ Department of Computer \\ Science and Engineering \\ BRAC University \\ 66 Mohakhali, Dhaka-1212, \\ Bangladesh
}

\author{
Fabian Parsia George \\ Department of Computer \\ Science and Engineering \\ BRAC University \\ 66 Mohakhali, Dhaka-1212, \\ Bangladesh
}

\author{
Mita Halder \\ Department of Computer \\ Science and Engineering \\ BRAC University \\ 66 Mohakhali, Dhaka-1212, \\ Bangladesh
}

\begin{abstract}
The significance of home security framework can't be denied in the present crime-ridden society. Home security is getting to be noticeably important these days as the potential outcomes of interruption are expanding day by day. Protection from burglary, leaking of crude gas and fire are the most vital prerequisites of home security systems for individuals. The most common system used for such purpose is CCTV camera. However, it is expensive, uses excessive space for keeping records and furthermore expects manpower to supervise the unapproved action. In this paper, we proposed a system which is cost effective with better resolution and can detect intrusion single-handedly. Here, PIR motion sensor, along with the Raspberry $\mathrm{Pi}$, is used to detect motion and once it is activated, a video is triggered via $\mathrm{Pi}$ camera module. IOT based applications can be used remotely to view image and get email on intrusion detection.
\end{abstract}

\section{General Terms}

Security, Intrusion Detection, Face Recognition.

\section{Keywords}

Motion Detection, Raspberry Pi, Camera Module, IOT, Facial Landmark, Email Notification.

\section{INTRODUCTION}

Home security has dependably been an issue in the present day. While we are adapting up to the pace of advancing technology and rising economy, our activities may leave our natural belonging in an unsecured and unsafe state. Stunning as it might come, yet an estimated 3.7 million thefts occurred on an average, every year from 2003 to 2007 [1]. 30 percent of these are a direct result of an open or opened window or entryway and 66 percent of all robberies are home break-ins [1]. Robberies and thefts have become one of the major problems in our daily life, and in such situation, the availability of a security system for our house and office has become an essential requirement in order to ensure our safety. Obviously, regardless of having such dangers, our day by day routine must be taken after. To make things less demanding, the majority of the population goes with conventional systems such as burglar alarm system that rings when somebody enters their room. An alternate solution will be a CCTV that keeps video records of the targeted area, that too is monitored by an authorized body [2, 3, 4]. However, configuring such systems in local residents can be strenuous and furthermore, once the burglary occurs, the notification to the householder is not spontaneous. In other words, the system is effective if the owner is near-by, but one can't make a move in due time if the case is inverse $[2,3,4]$. Thus, we are proposing a device that can notify you home-breach instantly via IOS/Android phone.

The resulting segments of the paper have been structured as follows: Section II includes the related work and the existing systems based on our proposed system. Section III illustrates a proposed model with block diagram and flowchart concerned with the working of the system. Section IV describes how the individual components of the system work. Section V represents the system implementation of our proposed model and section VI concludes the paper.

\section{RELATED WORK}

Ever since the wireless home security systems have become a highly developed research in the field of International Intelligent Building, various approaches were proposed for designing such systems. In [7], an LED is used to monitor the home entryway and once intrusion occurs, the system enables house owner to change the passkey for the entrance. In [8], on account of intrusion, the system notifies the owner by sending SMS to the owner's registered phone number that is already preconfigured in the system. The owner can also remotely control his home by SMS using only his registered number. In [9], a system is proposed that comprises of IR sensors to recognize intruder, Raspberry Pi to capture intruder's picture, and a GSM modem to send SMS alongside the hyperlink of the picture. In [10], a robot is used to transmit live streaming of the home that can be viewed with a smartphone. The robot notifies the owner, on account of an intrusion, with the help of a temperature sensor that is integrated within the robot. In [11], GSM technology is used to alert the owner via mail once an intrusion is detected. In [12], a system is introduced that includes a raspberry pi and a camera to capture information and send to a smartphone that is used to remotely observe a specific location.

\section{PROPOSED MODEL}

The proposed system has been intended to overcome the drawbacks of the past surveillance systems and to enhance the security, adaptability, efficiency at whatever point required. Having a security camera system may infrequently be unimaginable because of the thorough expenses brought about in the course of the installation. The objective of our system is to make a smart surveillance system which can provide caution to the owner remotely by means of notification. It does this by sending an email the owner's smartphone when an intrusion is detected. The intrusion is detected using PIR motion sensor and is activated as soon as a motion is detected. Once it is activated, it will trigger video recording via the Pi camera module. As the Pi camera starts recording video, it first cross-matches the face of the person who enters the room. If it's a familiar face, for example, that of a family member, then the detection is completely ignored. In case the face doesn't match, indicating the detection of an intruder, the security guard of that apartment is notified with the help of a buzzer to generate continuous beeps and an LCD to display on 


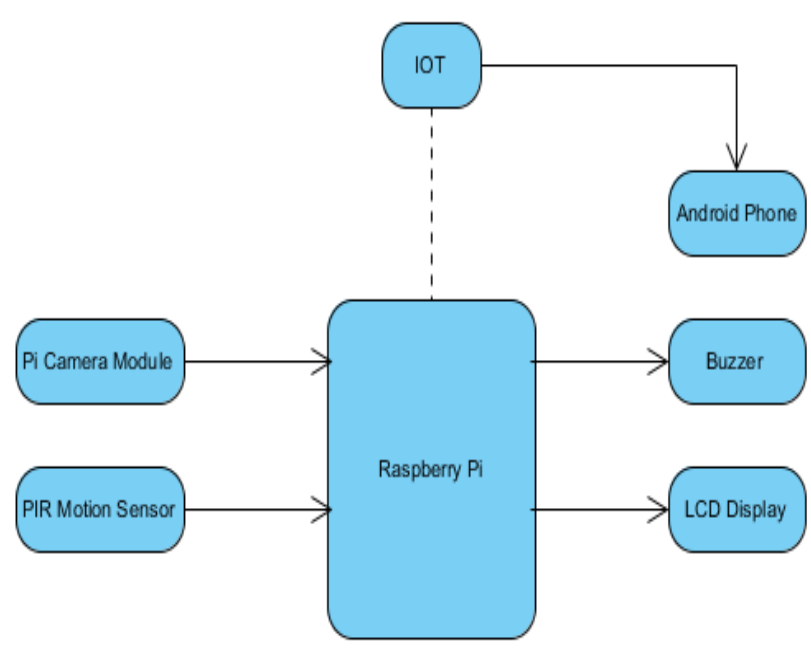

Fig 1: Block Diagram of the Proposed System

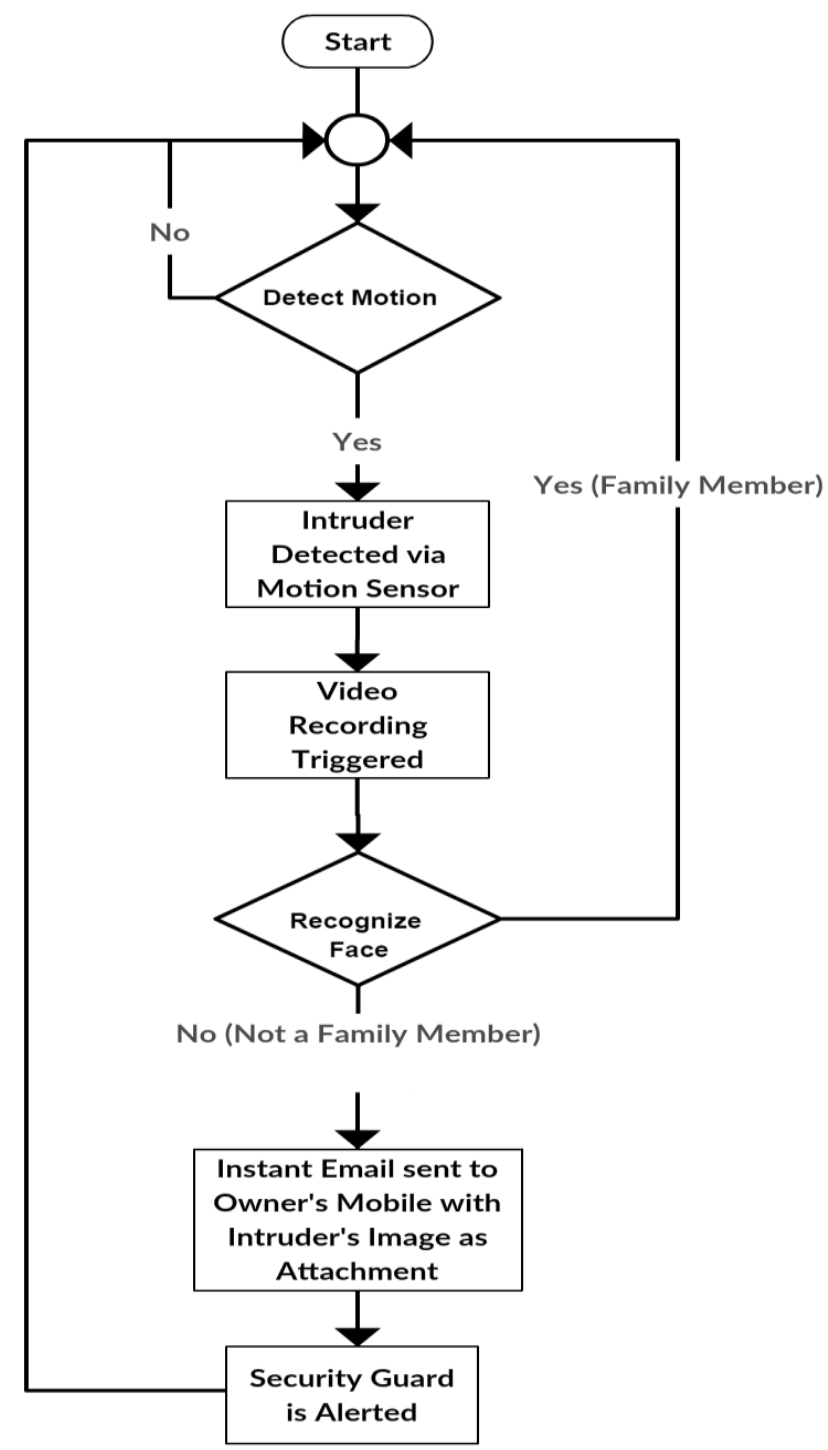

Fig 2: Flowchart of the Proposed System

which flat the intrusion has occurred. Furthermore, an image of the intruder is captured, instantly uploaded in cloud, and a notification is sent via an email on his phone for the owner to see who is inside his room. One interesting thing to note here is that the camera for recording video will be activated for short duration on an account of an intrusion and as soon as the time exceeds, it will be turned off. It will again be activated if another intrusion occurs but for a limited duration, thus making our system more efficient. Figure 1 and Figure 2 represent the block diagram and the flow chart of our proposed system.

\section{COMPNENT DESCRIPTION}

\subsection{Raspberry Pi}

The Raspberry $\mathrm{Pi}$ is a microprocessor that is designed for the Linux operating system. It is a fully functional computer that consumes low power and is expected to perform similar to that of a desktop computer. In our proposed system, Raspberry Pi 3 Model B is used with Raspbian OS integrated into it. It has a Broadcom BCM2387 System on a Chip (SoC), which includes Quad-Core ARM Cortex-A53 $1.2 \mathrm{GHz}$ processor, 1GB RAM, and 64-bit CPU. It comprises of a micro SD card slot that supports a 32 GB SD card, DSI display connector, 40-pin GPOI connector, CSI Camera connector, an Ethernet port, and a wireless LAN \& Bluetooth connectivity $[13,14]$.

\subsection{Pi Camera Module}

The Raspberry Pi camera module can take high-definition video along with still pictures and can support 1080p30, 720 p60, and VGA90 video modes. A 16-pin ribbon of $15 \mathrm{~cm}$ is integrated with the camera module and is assigned to the CSI port of the Raspberry Pi [15]. It is advantageous to use Raspberry Pi camera module as there are plenty of third-party libraries build for it. For our work, we have used 8.5megapixel camera module of version 2.1

\subsection{Passive Infrared (PIR) Motion Senor}

The passive Infrared sensor is used to detect the motion of a person. When someone comes in the range of the PIR sensor, the temperature of the room increases due to the body temperature of that person. This rise in temperature indicates the motion of an individual [16].

\subsection{Liquid Cristal Display (LCD)}

The LCD Display is cost-effective and is used to display numbers, character, and graphics at low power consumption [17].

\subsection{Buzzer}

The buzzer is used to generate beep sound when a voltage is supplied. For our proposed system, the buzzer is used as an alert to generate continuous beep when an intrusion occurs.

\section{WORKING PRINCIPLE}

\subsection{Detection of Motion}

In our proposed system, PIR sensors are placed in the rooms in order to detect the motion of a person. When someone comes in the range of the PIR sensor, the temperature of the room increases due to the body temperature of that person. This rise in temperature indicates the motion of an individual. This triggers the pi camera on which immediately starts recording video.

\subsection{Detection and Identification of Face}

The Raspberry Pi, along with the PIR sensor and the camera module, starts processing the captured video as soon as an intrusion occurs. The face detection and recognition process is carried out in 3 stages.

i. Constructing an image dataset using the face of the authorized members (family members). 
ii. Training the face recognizing classifier with the images in the dataset.

iii. Detecting faces in the captured images and comparing them with the trainer dataset.

At the very beginning, 10 images from different side views are collected from the live streaming video of each authorized member. These images were captures at different times of the day and with different facial expressions. All these images were saved in a separate folder.

Once that is done, a Haar Cascade classifier is used. Object Detection using Haar feature-based cascade classifiers is an effective object detection method. It is a machine learning based approach where a cascade function is trained from a lot of positive and negative images. It is then used to detect objects in other images. Here we will work with face detection. Initially, the algorithm needs a lot of positive images (images of faces) and negative images (images without faces) to train the classifier. Then we need to extract features from it. For this, Haar features shown in figure 3 image are used. They are just like our convolutional kernel. Each element is a solitary esteem acquired by subtracting sum of pixels under the white rectangle from sum of pixels under the black rectangle.
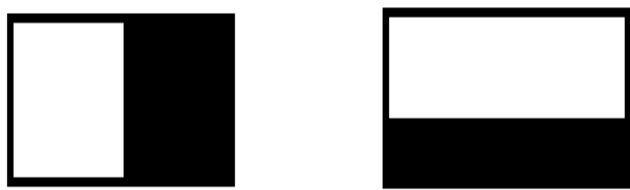

Fig 3: Edge Feature
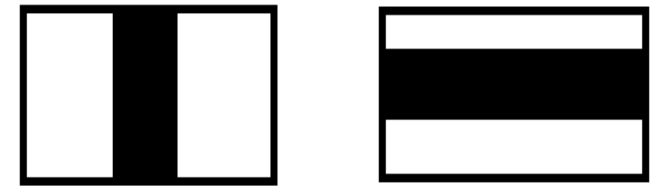

Fig 4: Line Feature

Now, all possible sizes and locations of each kernel are used to calculate lots of features. For each feature calculation, we need to find the sum of the pixels under white and black rectangles. To solve this problem, we used the integrated for of the picture. However substantial our picture is, it diminishes the computations for an offered pixel to a task including only four pixels they introduced the integral image. However For this, we apply each and every feature on all the training images. For each feature, it finds the best threshold which will classify the faces to positive and negative. Obviously, there will be errors or misclassifications. We select the features with minimum error rate, which means they are the features that most accurately classify the face and nonface images. In an image, most of the image is non-face region. So it is a better idea to have a simple method to check if a window is not a face region. If it does not find the face in a single attempt, it is not going to process the whole region. Instead, it will focus on regions where there can be a face. This way, we spend more time checking possible face regions. For this they introduced the concept of Cascade of Classifiers. Instead of applying all 6000 features on a window, the features are grouped into different stages of classifiers and applied one-by-one. In figure 5, we can see that if a window fails the first stage, discard it. We don't consider the remaining features on it. If it passes, apply the second stage of features and continue the process. The window which passes all stages is a face region.

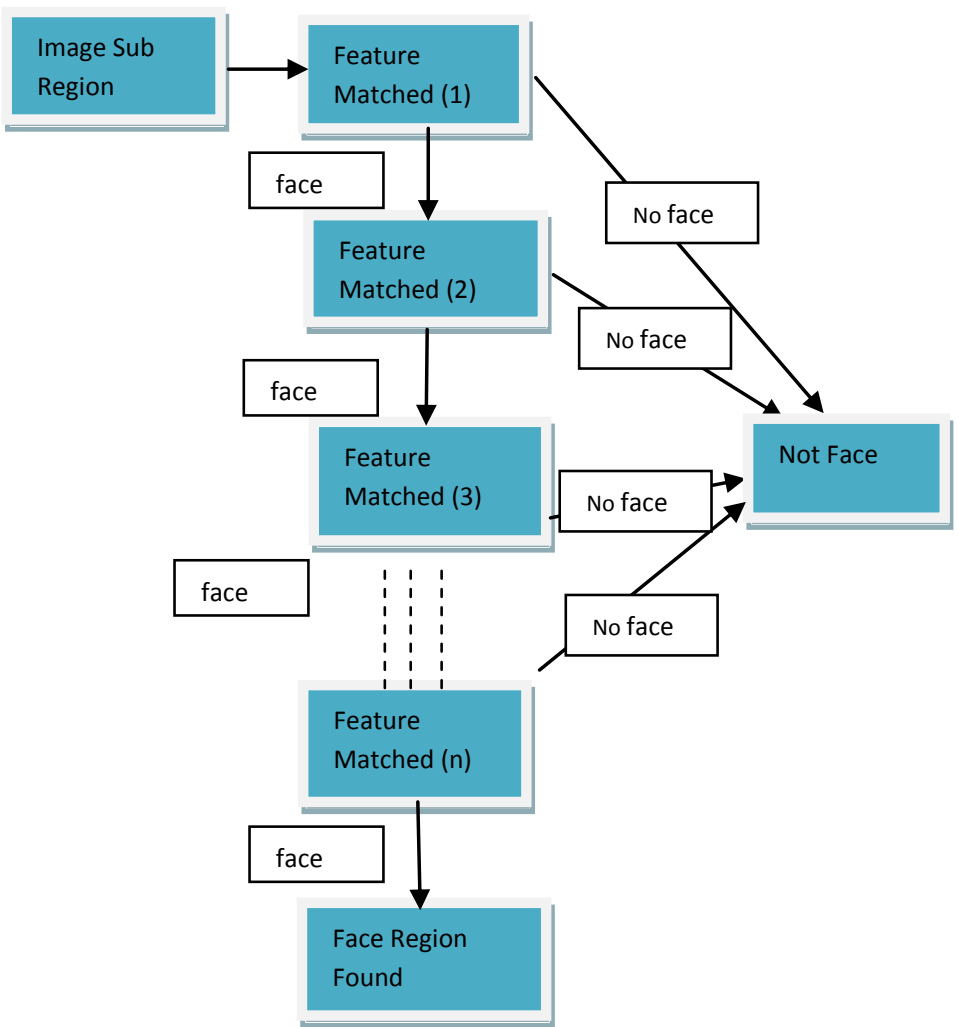

Fig 5: Finding Face from Different Sub region

Moreover, it uses this entire basic feature to detect the facial landmarks, such as the eyes, nose, and mouth, out of these images In order to do that, the classifier is first trained with AdaBoost and Haar feature algorithms that are implemented from the OpenCV Library [18]. The library is preconfigured with a pre-trained facial landmark detector which approximates the location of the $68(\mathrm{x}, \mathrm{y})$-coordinates that is related to the facial landmarks of the face [19]. After detected the facial landmarks of each face, the images are converted to a grayscale format to reduce the inherent complexity of the images $[20,21]$. To convert the image into grayscale we can use different approaches. Here we convert each pixel Red, Green and Blue value into Gray color.

$$
\begin{aligned}
\operatorname{Gray}(x, y)= & 0.2989 \times f(x, y, R)+0.5870 \times f(x, y, G)+ \\
& 0.1140 \times f(x, y, B)[25]
\end{aligned}
$$

where, $\operatorname{Gray}(\mathrm{x}, \mathrm{y})=$ Output image as grayscale image, $\mathrm{f}(\mathrm{x}, \mathrm{y}, \mathrm{R})$ $=$ Red channel pixel value in specific $(\mathrm{x}, \mathrm{y})$ coordinate, $\mathrm{f}(\mathrm{x}, \mathrm{y}, \mathrm{G})=$ Green channel pixel value in specific $(\mathrm{x}, \mathrm{y})$ coordinate and $\mathrm{f}(\mathrm{x}, \mathrm{y}, \mathrm{B})=$ Blue channel pixel value in specific $(\mathrm{x}, \mathrm{y})$ coordinate[25]. After converting the images into grayscale format, the trainer dataset in the library is used to store the formatted images of each authorized member along with a unique ID for identification

Once an intruder is detected, the 68 (x,y)-coordinates of his facial landmarks are extracted using the exact process and compared with the ones stored in the trainer dataset. If the coordinates are identical, indicating that the intruder is one of the authorized members, the system just displays his name but doesn't take any action. However, in case the coordinates differ, this indicates that the person is not one of the 
authorized members and thus the owner of the house must be notified via email.

\subsection{Notifying the Owner}

The Raspberry Pi is operated as a surveillance system where it sends email alerts to the owner on account of an intrusion, with the captured image as an attachment. The subject, message content, Email ID and the image of the intruder are all entered into the system through python scripts [22].

In order to send emails, Simple Mail Transfer protocol (SMTP) is used by implementing a native library in Python, smtblib. The smtplib module defines an SMTP client session object that can be used to send mail to any Internet machine with an SMTP or ESMTP listener daemon. MIME protocols are used to send attachments. SMTP connection is put in TLS (Transport Layer Security) mode to ensure security. Port number 587(for smtp.gmail server) is used for sending emails.

\subsection{Notifying the Security Guard}

Notifying the owner of the house is effective if he is near-by, but one can't take instant action if the case is opposite. Thus to enhance the level of security, the security guard of the apartment is notified as well so that he can make is move in due time. In order to notify the guard, a buzzer is used as an alert to generate continuous beep when an intrusion occurs. For him to acknowledge on which flat the intrusion has occurred, an LCD display is mounted somewhere in the security guard room which displays the suspicious flat number of the apartment.

\section{RESULTS AND DISCUSSION}

Constructing the trainer dataset required significant amount of time and attention. Initially, the pictures of an authorized member were taken in daylight in order to train the classifier. However, when carrying out the experiment at night, it was observed that the Haar Cascade classifier was unable to detect the member. As a result, an email notification was automatically sent to the owner, despite of him being a family member. The case was similar when still pictures of a family member were captured instead of capturing pictures from various angles and with different expressions. Taking all these parameters into consideration, we constructed a trainer dataset that can help the classifier to recognize the face of the intruder with better percentage accuracy.

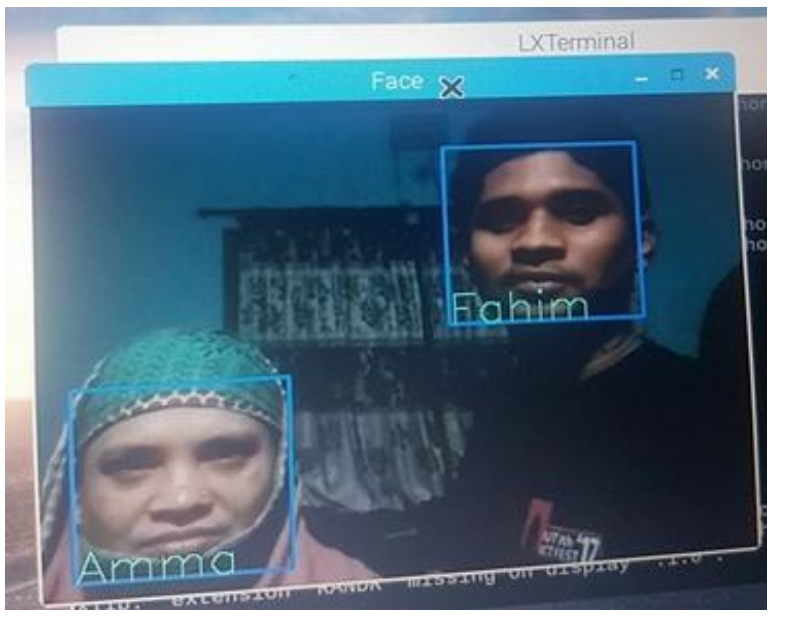

Fig 6: Face Recognition of the Authorized Member

On completion of this work, our system can successfully distinguish the between the family member and the intruder. Based in the comparison it can decide whether to ignore intrusion or to take further actions. The occurrence of intrusion is ignored when the image of the detected face is found to be matching with the images in the trainer dataset. In such case, the system only displays the name of the intruder who is none other than a family member. Figure 6 presents a snapshot of how the system could successfully recognize the face of the authorized member. On account of an intrusion, the security guard of the apartment is notified with the help of a buzzer and an LCD display and an email notification is also sent to the owner of the house. Figure 7 shows a snapshot of the owner's Gmail account after the email is sent.

\subsection{Performance Evaluation}

It was observed that the Raspberry Pi 3 Model B used in our proposed system could not perform fast image processing. As it was mentioned in section 4.1, the $\mathrm{Pi}$ is comprised of $1 \mathrm{~GB}$ RAM and 64-bit CPU, and with such specifications the system was not sufficiently fast. In order to improve the performance of the system, an attempt was taken to increase the clock speed. However, as the FPS (Frame per Second) rate of our video processing pipeline was very less, over-clocking the pi processor doesn't provide a decent outcome. As a result, multithreading was applied to significantly increase the FPS processing rate of our pipeline. Multithreading is a process through which a single core in a multi-core processor can perform multiple threads or processes at the same time [23].
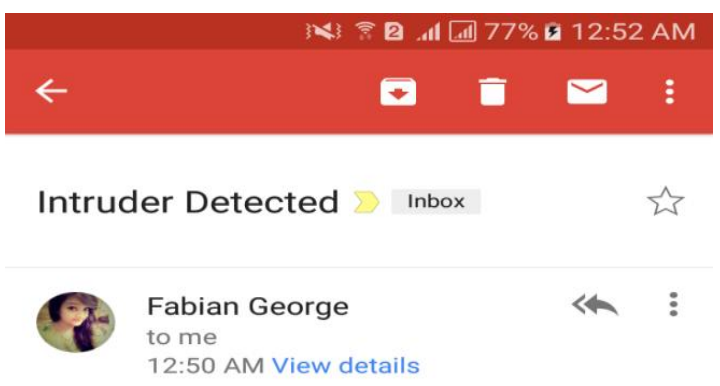

There is someone in your home, please check the attached picture

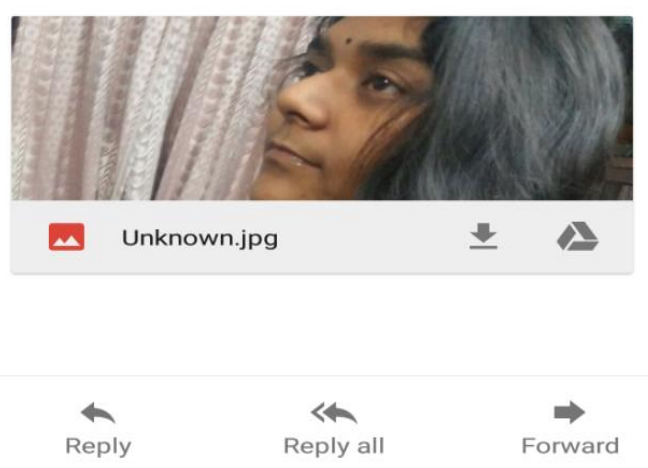

Fig 7: Result Displayed on Mobile through Email

Since the Pi 3 contains a Quad-Core processor, performing multi-threading makes our system more efficient in terms of speed. Table 1 and 2 illustrates the performance accuracy before and after executing multithreading. 
Table 1. Performance Accuracy before Executing Multithreading.

\begin{tabular}{|c|c|c|c|}
\hline $\begin{array}{c}\text { CPU Speed } \\
\text { (arm speed }) \\
(\mathbf{C C} / \mathbf{s})\end{array}$ & $\begin{array}{c}\text { GPU Speed } \\
(\mathbf{C C / s})\end{array}$ & $\begin{array}{c}\text { Frame per } \\
\text { Second }\end{array}$ & $\begin{array}{c}\text { Performance } \\
\text { Accuracy } \\
(\boldsymbol{\%})\end{array}$ \\
\hline 800 & 126 & 3 & 70 \\
\hline 1000 & 126 & 3 & 73 \\
\hline 1200 & 126 & 3 & 75 \\
\hline
\end{tabular}

Table 2. Performance Accuracy after Executing Multithreading.

\begin{tabular}{|c|c|c|c|}
\hline $\begin{array}{c}\text { CPU Speed } \\
\text { (arm speed) } \\
(\mathbf{C C} / \mathbf{s})\end{array}$ & $\begin{array}{c}\text { GPU Speed } \\
\text { (CC/s) }\end{array}$ & $\begin{array}{c}\text { Frame per } \\
\text { Second }\end{array}$ & $\begin{array}{c}\text { Performance } \\
\text { Accuracy } \\
(\boldsymbol{\%})\end{array}$ \\
\hline 800 & 126 & 4 & 78 \\
\hline 1000 & 126 & 6 & 83 \\
\hline 1200 & 126 & 7 & 88 \\
\hline
\end{tabular}

From Table 2, it can be deduced that, increasing the FPS rate using multithreading increased the performance accuracy of our proposed system by a significant amount. Therefore, it can be said that the application of multithreading can significantly improve the performance of image processing in Raspberry pi.

\section{CONCLUSION}

The urge of the smart security system for monitoring particular area is developing day by day in the recent years. The application of security system is uncountable as it can be used in various situations for different reasons. However, the traditional security systems such as the CCTV camera are quite expensive and require constant supervision. Taking all these issues into consideration, we have come up with a security surveillance that is capable of detecting intruders and taking appropriate actions against it. The system informs the authorized owner of an intrusion via mail except for the ones who are the authorized members, such as the family members. In addition, it will also alert the security guard of the building via a buzzer and display on which flat the intrusion has occurred through an LCD Display. The system is very beneficial for people who want to safeguard their properties and restrict access. It is very easily operated, so that anybody whether rich or comfortable, young or old can make use of this system. In the future, we would like to work on recognizing the pattern of the intruder for intrusion detection. The burglars, for example, have particular patterns common in them. This includes maintaining a pin drop silent after entering the house, the fear of getting caught that is visible in face, and body movements in search of valuable things that can be stolen. We believe, if these patterns can be detected and recognized, a near perfect surveillance system can be designed. Furthermore, as the need to be secured and safe is becoming a growing concern in the recent years, we would like to make our security system intelligent that will be both affordable and can be used in various areas such as swimming-pools, school precincts, and embassies.

\section{ACKNOWLEDGMENTS}

First and foremost, we take this opportunity to express our profound gratitude and deep regards to our theory faculty Dr.
Khalilur Rahman for his contribution in stimulating suggestions and encouragement throughout the course of our project. The blessing, help, and guidance given by him from time to time shall be carried by us a long way in the journey of life on which we are about to get on.

We also take this opportunity to express a deep sense of gratitude to our lab faculties Dr. Md. Ashraful Alam and Dr. Jia Uddin for their aspiring guidance, constant supervision, and friendly advice during the project work, which were extremely valuable, both theoretically and practically. Without their assistance and dedicated involvement in every step throughout the process, this project would have never been accomplished.

Finally, our thanks and appreciations also go to the BRAC University and the Department of Computer Science and Engineering (CSE), for appreciating the student's enthusiasm towards new innovations and for providing them the opportunities to develop such projects.

\section{REFERENCES}

[1] Shannan M. Catalano, "Victimization During Household Burglary," Bureau of Justice Statistics, National Crime Victimization Survey, 2010.

[2] X. Cai, "MPEG-4 over local area mobile surveillance system," IEE Symposium Intelligent Distributed Surveillance Systems, 2003.

[3] V. B. Saiz and F. Gallego, "GPU: Application for CCTV systems," 2014 International Carnahan Conference on Security Technology (ICCST), 2014.

[4] "Turn your Pi into a low-cost HD surveillance cam," Raspberry Pi, 14-Oct-2013. [Online]. Available: https://www.raspberrypi.org/blog/turn-your-pi-into-alow-cost-hd-surveillance-cam. [Accessed: 06-Mar-2018]

[5] A. Antony, "Live Streaming Motion Detection Camera Security System with Email Notification using Raspberry $\mathrm{Pi}$," IOSR Journal of Electronics and Communication Engineering, vol. 01, no. 01, pp. 142-147, 2016.

[6] H. V. Khodaskar and S. Mane, "Human Face Detection \& Recognition Using Raspberry Pi," Icsesd-2017, 2017.

[7] L. Siwen and L. Yunhong, "Design and Implementation of Home Automation System," 2008 International Symposium on Information Science and Engineering, 2008.

[8] M. S. H. Khiyal, A. Khan, and E. Shehzadi, "SMS Based Wireless Home Appliance Control System (HACS) for Automating Appliances and Security," Proceedings of the 2009 InSITE Conference, 2009.

[9] P. Vigneswari, V. Indhu, R. R. Narmatha, A. Sathinisha, and J. M. Subashini, "Automated Security System using Surveillance," International Journal of Current Engineering and Technology, vol. 5,No.2, pp. 882-884, Mar. 2015.

[10] V. Persis Priyanka, K. SudhakarReddy,"PIR based Security Home Automation System with Exclusive Video Transmission", International Journal of Scientific Engineering and Technology Research, Vol.4, No.18, pp.3316-3319, 2015.

[11] J. Bangali and A. Shaligram, "Design and Implementation of Security Systems for Smart Home 
based on GSM technology," International Journal of Smart Home, vol. 7, no. 6, pp. 201-208, 2013.

[12] S. Prasad, P. Mahalakshmi, A. J. C. Sunder , and R. Swathi, "Smart Surveillance Monitoring System Using Raspberry PI and PIR Sensor," International Journal of Innovative Research in Advanced Engineering(IJIRAE), vol. 4, no. 4, pp. 11-15, Apr. 2017.

[13] A. Industries, "Raspberry Pi 3 - Model B - ARMv8 with $1 \mathrm{G}$ RAM," adafruit industries blog RSS. [Online]. Available: https://www.adafruit.com/product/3055. [Accessed: 06-Mar-2018].

[14] "Raspberry Pi Software," Exploring Raspberry Pi, pp. 23-54, 2016

[15] "Camera Module," Camera Module - Raspberry Pi Documentation. [Online]. Available: https://www.raspberrypi.org/documentation/hardware/ca mera. [Accessed: 06-Mar-2018].

[16] "Passive infrared sensor," Wikipedia, 28-Feb-2018. [Online].

Available: https://en.wikipedia.org/wiki/Passive_infrared_sensor. [Accessed: 06-Mar-2018].

[17] "Liquid-crystal display," Wikipedia, 03-Mar-2018. [Online]. Available: https://en.wikipedia.org/wiki/Liquid-crystal_display. [Accessed: 06-Mar-2018].

[18] X. Zhao, X. Chai, Z. Niu, C. Heng, and S. Shan, "Context constrained facial landmark localization based on discontinuous Haar-like feature," Face and Gesture 2011,2011

[19] "Facial landmarks with dlib, OpenCV, and Python," PyImageSearch, 30-Apr-2017. [Online]. Available: https://www.pyimagesearch.com/2017/04/03/faciallandmarks-dlib-opencv-python. [Accessed: 06-Mar2018].

[20] B. Heisele, T. Poggio, and M. Pontil, "Face Detection in Still Gray Images,"Computer Science and Artificial Intelligence Lab (CSAIL), Jan. 2000.

[21] J. Lu and K. Plataniotis, "On conversion from color to gray-scale images for face detection," 2009 IEEE Computer Society Conference on Computer Vision and Pattern Recognition Workshops, 2009.

[22] "Tutorial: How to send an email with Python," Nael Shiab, 03-Oct-2015. [Online]. Available: http://naelshiab.com/tutorial-send-email-python/. [Accessed: 06-Mar-2018]

[23] [25] "Multithreading (computer architecture)," Wikipedia, 01-Mar-2018. [Online]. Available: https://en.wikipedia.org/wiki/Multithreading_(computer_ architecture). [Accessed: 20-Mar-2018].

[24] A. B. Forouzan, Data communications \& networking (sie). Tata McGraw-Hill Education, 2006.

[25] R. C. Gonzalez and R. E. Woods, Digital image processing (3rd Edition). Pearson Education International,2008 